\title{
ON A CLASSIFICATION OF PLANE DOMAINS FOR HARDY CLASSES
}

\author{
SHŌJI KOBAYASHI
}

\begin{abstract}
For every positive nubmer $p$, let $O_{p}$ denote the class of plane domains $W$ for which the Hardy class $H_{p}(W)$ contains no nonconstant functions, and $O_{p}^{-}=\bigcup\left\{O_{q}: 0<q<p\right\}$. In this paper it is proved that $O_{p}$ strictly contains $O_{p}^{-}$if $p>1$.
\end{abstract}

1. Introduction. Let $W$ be a domain in the extended complex plane $S$. For every positive number $p$, let $H_{p}(W)$ be the class of all single-valued analytic functions $f$ in $W$ for which $|f|^{p}$ admits a harmonic majorant there. Let $O_{p}$ denote the class of $W$ such that $H_{p}(W)$ contains no functions but the constants. Set $O_{p}^{-}=\cup\left\{O_{q}: 0<q<p\right\}$. In this paper we deal with the inclusion relations among these classes of plane domains. The corresponding problem for arbitrary Riemann surfaces is completely solved by Heins [2]. For plane domains, however, the best results that we know by now are the following, one of which is due to Hejhal [4], and the other to the author [5]:

Theorem A (Hejhal). For $n=2,3, \ldots$,

$$
O_{n / 2}^{-}<O_{n / 2} \text {. }
$$

TheOREM B (KobAYASHI). For $n=2,3, \ldots$, and $p>n / 2$,

$$
O_{n / 2}<O_{p}
$$

Here $<$ means a strict inclusion relation.

The object of this paper is to prove the following theorem, which evidently includes the above two.

MaIN TheOREM. If $p \geqslant 1$, then

$$
O_{p}^{-}<O_{p}
$$

CoROLlaRY. If $p \geqslant 1$ and $q<p$, then

$$
O_{q}<O_{p}
$$

The Corollary is an easy consequence of the Main Theorem, since $O_{q} \leqslant$ $O_{p}^{-}$if $q<p$.

The idea of this paper was partially motivated by that of Hejhal [3], [4]. I would like to express my sincere thanks to Professor N. Suita for his

Received by the editors February 10, 1977 and, in revised form, June 9, 1977.

AMS (MOS) subject classifications (1970). Primary 30A78, 30A48.

Key words and phrases. Hardy class, harmonic majorant, subharmonic function, superharmonic function. 
encouragement and helpful suggestions.

2. Hejhal's lemmas. In this section we state two lemmas, which were proved by Hejhal [4, Theorems 10 and 17]. As for Lemma 2, however, we give another proof which is more elementary than that of Hejhal's and essentially due to Shimbo [6].

LeMMA 1. Let $c_{1}, \ldots, c_{m}$ be positive numbers with $c_{1}+\cdots+c_{m}=2$, $m>2$. Suppose that $E$ is a compact totally disconnected set of linear measure 0 which lies on an $m$-star formed by $m$ rays emanating from the origin to the point at infinity, with successive angles $\pi c_{1}, \ldots, \pi c_{m}$. Let $c_{0}=\max \left\{c_{j}: j=\right.$ $1, \ldots, m\}$ and $p \geqslant 1$. Suppose that $f \in H_{p}(S-E)$; then

$$
f(z)=\sum_{\nu=0}^{\infty} a_{\nu} z^{-\nu} \quad(0<|z|<\infty),
$$

with $a_{\nu}=0$ for all $\nu \geqslant 1 / p c_{0}$.

For the proof see Hejhal [4, pp. 15-18].

LEMMA 2. There exists a compact totally disconnected set $F$ such that (i) $F$ lies on the real axis; (ii) $F$ is symmetric with respect to the origin; (iii) $F$ is of linear measure 0 ; and (iv) $z^{-1} \in H_{p}(S-F)$ for all $p$ with $0<p<1$.

Proof. Let $A$ be a compact totally disconnected set such that (a) $A$ lies on the interval $[-1,1]$; (b) $A$ is symmetric with respect to the origin; (c) $0 \notin A$; (d) $A$ is of linear measure 0 ; and (e) $A$ is of logarithmic capacity positive. For example, we can take the Cantor ternary set. Let $B=\cup_{k=-\infty}^{\infty} A+2 k$ and $F_{1}$ be the image of $B$ under the map $h(z)=z^{-1}$. Finally let $F=F_{1} \cup\{0\}$. We shall prove that $F$ satisfies (i), .., (iv) of the lemma. All except (iv) are trivial. Since $h$ maps $S-F$ conformally onto $C-B$, we must show that $z \in H_{p}(\mathrm{C}-B)$ for all $p$ with $0<p<1$. It is well known that every analytic function $f$ for which $|\operatorname{Im} f|$ admits a harmonic majorant is of class $H_{p}$ for all $p$ with $0<p<1($ see $[1, \mathrm{p} .35])$. Therefore it is sufficient to prove that $|\operatorname{Im} z|$ admits a harmonic majorant in $\mathbf{C}-B$. Let $\omega$ be the harmonic function in $(C-B) \cap\{z: \operatorname{Im} z<2\}$ with boundary value 1 on the line $\{z: \operatorname{Im} z=2\}$ and 0 on $B$. Since $\operatorname{Cap}(B)>0$, we see that $\omega$ is nonconstant. Since $B$ is invariant under the translation $\phi(z)=z+2$, so is $\omega$. Hence,

$$
\begin{aligned}
\sup \{\omega(z): \operatorname{Im} z=1\} & =\sup \{\omega(z): \operatorname{Im} z=1 \text { and }-1 \leqslant \operatorname{Re} z \leqslant 1\} \\
& <1-\varepsilon
\end{aligned}
$$

for some $\varepsilon$ with $0<\varepsilon<\frac{1}{2}$. Therefore

$$
\varepsilon^{-1} \omega(z)<\operatorname{Im} z+\varepsilon^{-1}-2
$$

on the line $\{z: \operatorname{Im} z=1\}$ and, hence, in $\{z: 1<\operatorname{Im} z<2\}$ by the maximum principle. We define $s$ in $\mathbf{C}-B$ as follows:

$$
s(z)= \begin{cases}\operatorname{Im} z+\varepsilon^{-1} & \text { if } \operatorname{Im} z \geqslant 2, \\ \varepsilon^{-1} \omega(z)+2 & \text { if } \operatorname{Im} z<2 .\end{cases}
$$


We shall show that $s$ is superharmonic in $\mathbf{C}-\boldsymbol{B}$. To show this, it is sufficient to prove that $s$ is superharmonic on the line $\{z: \operatorname{Im} z=2\}$. But this follows easily from (5) (see the proof of Lemma 3 below). Then we see that $s(z)+s(\bar{z})$ serves as a superharmonic majorant of $|\operatorname{Im} z|$ in $\mathbf{C}-\boldsymbol{B}$. Since $|\operatorname{Im} z|$ is subharmonic in $\mathbf{C}-\boldsymbol{B}$, it admits a harmonic majorant there, as desired.

3. Proof of the Main Theorem. We must construct a plane domain $W$ such that $W \in O_{p}-O_{p}^{-}$. Let $F$ be as in Lemma 2, and $\psi$ be the conformal map of $\{\zeta:|\arg \zeta|<\pi\}$ onto $\{z:|\arg z|<\pi / p\}$ defined by the branch of $z=$ $\zeta^{1 / p}$ such that $\psi(1)=1$. Let $E_{0}$ be the image of $F \cap\{\zeta: \operatorname{Re} \zeta>0\}$ under $\psi$. Let $c_{1}, \ldots, c_{m}$ be positive numbers with $c_{1}+\cdots+c_{m}=2, m \geqslant 2$, such that $c_{0}=\max \left\{c_{j}: j=1, \ldots, m\right\}=1 / p$. Let $E_{1}=\cup_{\nu=1}^{m} e^{i \theta_{v}} E_{0}$, where $\theta_{\nu}=$ $\pi \sum_{j=1}^{\nu} c_{j}$. Finally, let $E=E_{1} \cup\{0\}$ and $W=S-E$. On applying Lemma 2, it turns out that $H_{p}(W)$ contains no nonconstant functions, i.e., $W \in O_{p}$.

Next we must show that $H_{q}(W)$ contains a nonconstant function for any $q<p$. Let $q$ be fixed with $0<q<p$. We shall prove that $f(z)=z^{-1}$ belongs to $H_{q}(W)$. Let $w(z)=|z|^{-q}$. We must show that $w$ admits a harmonic majorant in $W$. Let $\lambda$ be the least harmonic majorant of $w_{1}(\zeta)=$ $|\zeta|^{-q / p}$ in $S-F$. We define $\chi$ in $W$ as follows:

$$
\chi(z)=\lambda\left(\psi^{-1}\left(e^{-i \theta_{\nu}}\right)\right)+g(z) \text { if } \theta_{\nu}-\frac{\pi}{2} c_{\nu} \leqslant \arg z<\theta_{\nu}+\frac{\pi}{2} c_{\nu+1}
$$

for $\nu=1, \ldots, m$, where $c_{m+1}=c_{1}$, and $g$ is the Green's function of $W$ with pole at $\infty$. We shall show that $\chi$ serves as a superharmonic majorant of $w$ in $W$. It is trivial that $\chi \geqslant w$ in $W$ and that $\chi$ is superharmonic in $W$ except on the $m$-star $\left\{z: \arg z=\theta_{\nu}-(\pi / 2) c_{\nu}, \nu=1, \ldots, m\right\}$. The following lemma shows the superharmonicity of $\chi$ on the $m$-star.

Lemma 3. Let $0<\theta<\pi$. Define

$$
\mu(z)= \begin{cases}\lambda(z) & \text { if } 0<\arg z \leqslant \theta / 2, \\ \lambda\left(e^{-i \theta} z\right) & \text { if } \theta / 2<\arg z<\theta ;\end{cases}
$$

then $\mu$ is superharmonic on the line $\{z: \arg z=\theta / 2\}$.

ProOF. First we shall prove that

$$
\mu \leqslant \lambda \text { in }\{z: 0<\arg z<\theta\} .
$$

Let $\left\{I_{k}\right\}$ be a decreasing sequence of compact sets on the real axis such that (a) $I_{k}$ is a union of a finite number of closed intervals; (b) $I_{k}$ is symmetric with respect to the origin; (c) $I_{k} \supset F$ and (d) $F=\cap I_{k}$. Let $\lambda_{k}$ be the least harmonic majorant of $w_{1}$ in $S-I_{k}$. It is well known that $\lambda_{k}$ converges to $\lambda$ uniformly on every compact subset of $S-F$. Let

$$
\phi_{k}(z)=\lambda_{k}(z)-\lambda_{k}\left(e^{-i \theta_{z}}\right)
$$

then $\phi_{k}$ is harmonic in $S-\left(I_{k} \cup e^{i \theta} I_{k}\right)$. For every $x \in I_{k}$, we easily see

$$
\phi_{k}(x)=\lambda_{k}(x)-\lambda_{k}\left(x e^{-i \theta}\right)=w_{1}(x)-\lambda_{k}\left(x e^{i \theta}\right) \leqslant 0
$$


and

$$
\phi_{k}\left(x e^{i \theta}\right)=\lambda_{k}\left(x e^{i \theta}\right)-\lambda_{k}(x)=\lambda_{k}\left(x e^{i \theta}\right)-w_{1}(x)=-\phi_{k}(x),
$$

since $\lambda_{k}$ is symmetric with respect to the real axis. By symmetry, $\phi_{k}=0$ on the 4-star $\{z: \arg z=\theta / 2+j \pi / 2, j=0,1,2,3\}$. Applying the maximum principle, we see that $\phi_{k} \geqslant 0$ in $\{z: \theta / 2<\arg z<\theta / 2+\pi / 2\}$. By letting $k \rightarrow \infty$, we get (7).

Let $z_{0}$ be any point with $\arg z_{0}=\theta / 2$, and let $r$ be a small positive number. Using (7) we see that

$$
\begin{aligned}
\mu\left(z_{0}\right) & =\lambda\left(z_{0}\right)=\frac{1}{2 \pi} \int_{0}^{2 \pi} \lambda\left(z_{0}+r e^{i \alpha}\right) d \alpha \\
& \geqslant \frac{1}{2 \pi} \int_{0}^{2 \pi} \mu\left(z_{0}+r e^{i \alpha}\right) d \alpha,
\end{aligned}
$$

and, hence, $\mu$ is superharmonic at $z_{0}$ as desired.

ADDED IN PROOF. Recently M. Hasumi [7] has completely solved the $H_{p}$ classification problem for plane domains, which we treated in this paper, in more general form than Heins [2] solved the problem for arbitrary Riemann surfaces.

\section{REFERENCES}

1. P. L. Duren, Theory of $H_{p}$ spaces, Academic Press, New York, 1970.

2. M. Heins, Hardy classes on Riemann surfaces, Lecture Notes in Math., vol. 98, SpringerVerlag, Berlin and New York, 1969.

3. D. A. Hejhal, Classification theory for Hardy classes of analytic functions, Bull. Amer. Math. Soc. 77 (1971), 767-771.

4. Classification theory for Hardy classes of analytic functions, Ann. Acad. Sci. Fenn. Ser. A I No. 566 (1973), 1-28.

5. S. Kobayashi, On $H_{p}$ classification of plane domains, Kōdai Math. Sem. Rep. 27 (1976), 458-463.

6. T. Shimbo, On harmonic majoration, Kōdai Math. Sem. Rep. 28 (1977), 278-283.

7. M. Hasumi, Hardy classes on plane domains, preprint, Inst. Mittag-Leffler Rep. 2 (1977) (to appear).

Department of Mathematics, Tokyo Institute of Technology, Tokyo, Japan 